\title{
Samples and data accessibility in research biobanks: an explorative survey
}

Marco Capocasa, Paolo Anagnostou, Flavio D'Abramo, Giulia Matteucci, Valentina Dominici, Giovanni Destro Bisol, Fabrizio Rufo Biobanks, which contain human biological samples and/or data, provide a crucial contribution to the progress of biomedical research. However, the effective and efficient use of biobank resources depends on their accessibility. In fact, making bio-resources promptly accessible to everybody may increase the benefits for society. In fact, optimizing their use and ensuring their quality will promote scientific creativity and, more in general, contribute to the progress of bio-medical research. Although this has become a rather common belief, several laboratories are still secretive and withhold samples and data. In this study, we conducted a questionnaire-based survey in order to investigate sample and data accessibility in research biobanks operating all over the world. The survey involved a total of 46 biobanks. Most of them gave permission to access their samples (95.7\%) and data (85.4\%), but free and unconditioned accessibility seemed not to be common practice. The analysis of the guidelines regarding the accessibility to resources of the biobanks that responded to the survey highlights three issues: (i) the request for applicants to explain what they would like to do with the resources requested; (ii) the role of funding, public or private, in the establishment of fruitful collaborations between biobanks and research labs; (iii) the request of co-authorship in order to give access to their data. These results suggest that economic and academic aspects are involved in determining the extent of sample and data sharing stored in biobanks. As a second step of this study, we investigated the reasons behind the high diversity of requirements to access biobank resources. The analysis of informative answers suggested that the different modalities of resource accessibility seem to be largely influenced by both social context and legislation of the countries where the biobanks operate. 


\section{Samples and data accessibility in research biobanks: an explorative survey}

Marco Capocasa $^{1 *}$, Paolo Anagnostou ${ }^{1,2^{*}}$, Flavio D’Abramo ${ }^{3 *}$, Giulia Matteucci ${ }^{1}$, Valentina Dominici $^{1,2}$, Giovanni Destro Bisol ${ }^{1,2}$ and Fabrizio Rufo ${ }^{1,2}$

${ }^{1}$ Istituto Italiano di Antropologia, Rome, Italy

${ }^{2}$ Department of Environmental Biology, Sapienza University of Rome, Rome, Italy

${ }^{3}$ Charité Comprehensive Cancer Center, Berlin, Germany

* These authors have equally contributed to the work

11

12

13

14 Corresponding author:

15

16

17 Istituto Italiano di

19 phone number: 00390649912725

20 e-mail: marco.capocasa@uniroma1.it

21

22 


\section{Abstract}

24 Biobanks, which contain human biological samples and/or data, provide a crucial contribution to 25 the progress of biomedical research. However, the effective and efficient use of biobank 26 resources depends on their accessibility. In fact, making bio-resources promptly accessible to 27 everybody may increase the benefits for society. In fact, optimizing their use and ensuring their 28 quality will promote scientific creativity and, more in general, contribute to the progress of bio29 medical research. Although this has become a rather common belief, several laboratories are still 30 secretive and withhold samples and data. In this study, we conducted a questionnaire-based survey in order to investigate sample and data accessibility in research biobanks operating all over the world. The survey involved a total of 46 biobanks. Most of them gave permission to access their samples $(95.7 \%)$ and data $(85.4 \%)$, but free and unconditioned accessibility seemed not to be common practice. The analysis of the guidelines regarding the accessibility to resources of the biobanks that responded to the survey highlights three issues: (i) the request for applicants to explain what they would like to do with the resources requested; (ii) the role of funding, public or private, in the establishment of fruitful collaborations between biobanks and research labs; (iii) the request of co-authorship in order to give access to their data. These results suggest that economic and academic aspects are involved in determining the extent of sample and data sharing stored in biobanks. As a second step of this study, we investigated the reasons behind the high diversity of requirements to access biobank resources. The analysis of informative answers 42 suggested that the different modalities of resource accessibility seem to be largely influenced by both social context and legislation of the countries where the biobanks operate. 


\section{Introduction}

47 Biobanks play a crucial role in the biological research involving human subjects and provide a

48 fundamental contribution to the rapid growth of scientific endeavour. This has been well

49 demonstrated, particularly in the past ten years, by the investments made by many countries in

50 order to build such infrastructures and to manage the biological resources they store (Kaye,

51 2011). Biobanks hold human biological samples and/or data to facilitate research over time

52 (Wolf et al., 2012). Their development across the world, together with improvements in

53 laboratory technologies, have dramatically increased opportunities to study collections of bio-

54 specimens (and their related data) with broader perspectives than those possible by small

55 collections maintained by single research groups (Haga \& Beskow, 2008). However, in addition

56 to the creation of these new opportunities, the rapid evolution taking place in the biobanking

57 field has created new challenges for researchers due to the huge potential benefits of having

58 access to biological resources.

59 In order to draw a more detailed picture of how biobanks manage their resources, as well as

60 considering the relationships (and even the contradictions) between the material and the

61 informational spheres of biological samples, we must take into account the propensity of these

62 institutions to share bio-specimens and data across scientific communities. The first challenge for

63 biobanks consists in finding an equilibrium between the scientific interests of researchers and the

64 expectations of donors. This can be reached by better exploiting the capabilities and flexibility of

65 current forms of informed consent (Kaye, 2012; Macilotti, 2013; Colledge et al., 2014;

66 D'Abramo, 2015). However, the design of an informed consent able to guarantee the

67 sustainability of resource availability does not solve the issues related to the economic interests

68 usually hidden behind the scientific research. This is the case of several web services that sell 
69 direct-to-consumer genetic tests. Through their activities, these companies accumulate large 70 amounts of samples and data that however, remain unavailable to most research communities 71 and groups (e.g. deCODEme, 23andme, Navigenics; see Knoppers, 2010). Finally, even if 72 biobanks embrace the open science principles, many bioethical issues can emerge as sample and 73 data sharing policies are different from country to country. In fact, the existence of local

74 legislation ensures compliance with habits and values characterizing the socio-cultural contexts 75 in which biobanks operate (Kaye, 2006; Haga \& Beskow, 2008). On the other hand, a 76 widespread and efficient sharing of bio-resources from different countries can only be assured 77 through the achievement of a global consensus on the legislation, the standards and the modalities to be followed. Starting from the preparation of informed consent, the biobank staff must take into account a number of issues when planning the management of the samples and data. They have to meet the requirements imposed by ethics committees, overcome the

81 difficulties in explaining the future uses of existing samples and put the potential donor in a 82 condition that will allow him to make a really informed decision (Colledge et al., 2014; 83 D’Abramo, Schildmann \& Vollmann, 2015).

84 Given these premises, it cannot be denied that the progress of human biological research largely 85 depends on the openness of resources and scientific knowledge. In fact, making bio-resources 86 promptly accessible to everyone could favour the common good. Furthermore, optimizing their 87 use, controlling quality and promoting general scientific creativity will guarantee a more rapid 88 and efficient progress of bio-medical research (Fischer \& Zigmond, 2010; Trinidad et al., 2010; 89 Milia et al., 2012; Oliver et al., 2012). Although this has become a rather common belief, several 90 laboratories are still secretive and withhold samples and data (Nelson, 2009; Cadigan et al., 91 2014). The scientific and academic interests of researchers are important, but they also have 
92 responsibilities towards the tax paying public. In fact, the scientific community often regards

93 biobanks and their services as simple source of material for the research and forget that the

94 sample come from human subjects. Milanovic, Pontille and Cambon-Thomsen (2007) have

95 clarified this concept, defining biobanks as "ambiguous entities" that "might be presented as

96 places for archival storage of a cultural patrimony freely accessible for relevant activities, or as

97 commercial enterprises with lucrative potential". At the same time, biobank donors have also

98 raised concerns about the fact that, in particular conditions, private and commercial interests in

99 biobanking may prevail over the public good and this could lead to social tensions (Godard et al.,

100 2010). The importance of identifying solutions which satisfy the needs of both researchers and

101 citizens is well testified by the engagement of a political economic structure such as the

102 Organisation for Economic Co-operation and Development (OECD) in supporting open access

103 to public funded research products (Arzberger et al., 2004).

104 Previous studies conducted on European and U.S. biobanks have provided information on the

105 developing trends of biobanking, giving detailed pictures of the type of sample and data stored

106 therein (Hirtzlin et al., 2003; Zika et al., 2011; Henderson et al., 2013). Other studies have

107 investigated the opinion of participants and the public about the relationships between sample

108 and data sharing practices and privacy concerns (Kaufman et al., 2009; Lemke et al., 2010).

109 However, to date, only a limited number of studies have faced the issue of sample and data

110 sharing behaviour of research biobanks (e.g. see Milanovic, Pontille \& Cambon-Thomsen, 2007;

111 Pereira, 2013). The present work aims to investigate sample and data accessibility in research

112 biobanks operating all over the world by means of a questionnaire-based survey. We observed a

113 low rate of free accessibility for both data and biological samples while the requirements for

114 accessing to the non-open resources were found to be highly heterogeneous. In order to evaluate 
115 the reasons behind this heterogeneity, we analysed the relationships between sharing strategies

116 and legal frameworks of the countries in which biobanks operate.

\section{Materials \& Methods}

119 In this study, we considered the definition of "biobank" as a repository that stores human

120 biological samples, with or without linking them to genetic or clinical data (see Haga \& Beskow,

121 2008). Therefore, we have not taken into account non-human bio-repositories or on-line

122 databases. The online survey was administered to a total of 238 biobanks (see Table 1) operating

123 in Europe (95), America (104), Asia (25), Africa (2) and Oceania (12). The biobanks were

124 selected in February 2014, through the use of three keywords ("biobank", "research biobank"

125 and "human biobank") and three search engines (Google, Google Scholar and Pubmed). Firstly,

126 we used the generic keyword "biobank" obtaining around 550,000 results with Google, 25,000

127 with Google Scholar and 2,100 with Pubmed. To refine our search, we added the term "research"

128 and obtained around 16,700 results with Google, 428 with Google Scholar and 25 with Pubmed.

129 Furthermore, we performed a second refinement adding the term "human" and obtained 4,500

130 results with Google, 284 with Google Scholar and 19 with Pubmed. We than inspected all these

131 latter results and identified the ones that refer to research biobank sites from which we recorded

132 their contact emails. This keyword-based procedure was adopted in order to select a random

133 sample of biobanks that could be easily found by anyone (researchers and the public).

134 The questionnaire was compiled in order to obtain a detailed picture of the sampling activities,

135 the sample and data accessibility criteria and the legal frameworks for their access. The final part

136 of the questionnaire was based on a preliminary analysis of twenty biobanks selected following a

137 geographic criterion (9 European, 3 North-American, 2 South-American, 3 Asian and 3 
138 Australian biobanks). The preliminary analysis was conducted by contacting each biobank

139 asking for explanations regarding their sample and data sharing modalities. We asked them to

140 provide information replying to our e-mail and/or by unstructured telephone interviews. Five

141 biobanks responded to our request and with two of them, we also conducted the interview.

142 Furthermore, we analysed their web sites in order to verify the presence of specific information

143 about these aspects. Finally, we used the collected information to build the questionnaire of the

144 present study. The questionnaire consisted of 21 questions (9 closed and 12 open-ended)

145 organized into three sections (see Supplemental File S1). The first section (General information)

146 refers to the name and the place where the biobank operates and other information regarding

147 funds, the sampling criteria adopted and the type of biological resources stored (sample and/or

148 data). The second section (Biological samples) investigates the sample collection, the ethical

149 requirements and the legal framework to which the biobank refers to for the management of

150 accessibility to biological samples. The last section (Data) includes questions regarding the data

151 collection and the legal framework to which the biobank refers to for the regulation of data

152 accessibility. The questionnaire was built and administered using Google Forms

153 (http://www.google.com/forms/about/) and survey participation was requested by contacting

154 each respective biobank address by e-mail, explaining the aims of our research. We launched our

155 survey on $18^{\text {th }}$ April 2014 and sent three reminders (28 ${ }^{\text {th }}$ April, $5^{\text {th }}$ May and $19^{\text {th }}$ May, 2014),

156 closing it at the end of May 2014. As previously stated in the invitation form, the administration

157 of the questionnaire was carried out anonymously. Neither personal information nor the names of

158 biobank were disclosed to others in managing the dataset.

159 The descriptive statistics of the answers to the closed questions were obtained using Microsoft

160 Excel 2010. Open questions were analysed considering the clarity and informative nature of the 
161 answers subdividing them into three categories: exhaustive answer (it provides a clear and

162 complete explanation of the question); partial answer (some information is missing); elusive or

163 no answer (it does not provide any of the information requested). Furthermore, since many of the

164 answers provided links to external resources (e.g. web links), we also evaluated the

165 exhaustiveness of these documents in order to acquire the information needed to fulfil the

166 questions. When the external references failed to provide clear information, depending on

167 retrievability difficulties or language issues (the replies were written neither in English nor in

168 Italian), we classified the answer as partial or elusive. Data was uploaded as online supporting

169 information (Supplemental File S2) and deposited in Zenodo (DOI: 10.5281/zenodo.17098).

171

172 Results

173

174

General information of the responded biobanks

175 Overall, we obtained responses from 46 out of 238 biobanks (19.3\%): 26 in Europe, 16 in

176 America, 2 in Asia and 2 in Oceania (Table 1). Most of the participant institutions are from

177 United States $(30.4 \%, \mathrm{n}=12)$ followed by the United Kingdom $(13.0 \%, \mathrm{n}=6)$, Italy $(10.9 \%, \mathrm{n}=5)$

178 and Germany $(6.5 \%, n=3)$.

179 More than half of the 46 biobanks are publicly funded (58.7\%), whereas $23.9 \%$ and $17.4 \%$ make

180 use of private (both profit-making and non-profit-making) funds or both types of funds,

181 respectively. Interestingly, some continental variations may be observed. In Europe, the rate of

182 institutions that receive only public funds is three times higher than that observed on the

183 American continent $(73.1 \%$ and $25.0 \%$ respectively) whereas the percentage of biobanks that 
184 make use of both types of funding is not significantly different ( $19.2 \%$ and $18.7 \%$, respectively).

185 All the Asian and Australian biobanks analysed here are only publicly funded. However, the low 186 number of institutions that responded to our survey (only 4) makes any form of comparison with

187 the other continents difficult.

188 Looking at the sampling criteria used by biobanks to collect bio-specimens, most of them

189 focused their attention only on disease-based samples $(41.3 \%)$ or coupling it with other criteria 190 such as type of tissue (17.4\%) or the geographic area where the samples were collected $(8.7 \%)$.

191 Only seven biobanks focus their attention on criteria other than diseases. Three consider 192 geography $(6.5 \%)$, two types of tissue $(4.3 \%)$, and two institutions concentrate on a population193 based-sample collection (4.3\%).

194 Regarding storing activities, a wide range of biological materials have been collected by the 195 sampled biobanks (e.g. blood, plasma, serum, urine, saliva, nucleic acids, cell lines). Eight

196 institutions store only bio-specimens and operate in the United States (3), in Europe (3; Italy, 197 Sweden and United Kingdom) in Asia (2). The remaining 38 biobanks store both biological 198 samples and data (89.1\%).

Bio-specimens collection and accessibility: legal and ethical aspects

201 In the first open question, we asked for the ethical requirements followed by the biobank for the 202 sample collection procedures (Question B2; see Figure 1). We mostly focused on the consent 203 obtained from participant (if any) and on approval by a third party [e.g. Ethics Committee, 204 Institutional Review Board (IRB)]. Twenty-two biobanks (47.8\%) provided informative answers, 205 referring, in all the cases, to the consent procedures and often pointing to guidelines, specific 206 local or international laws, and approval by ethics committees or institutional review boards. 
207 Open consent (through which participants give authorization to researchers for a broad range of

208 projects) seems to be the most utilized approach to involve donors. On the other hand, informed,

209 specific consent (in which participants authorize single projects whose aims, benefits and risks

210 should be well described, and through which biobanks should ask participants to give permission

211 again for every new project involving their samples and/or data) was found to be be frequent.

212 Some biobanks provide information on privacy issues describing, in most cases, the anonymized

213 characteristics of the personal data and the fact that they comply with national and federal laws

214 on data protection. Very few answers stressed the possibility for donors to pull out of the

215 biobank research (opt-out option). We categorized 15 answers (32.6\%) as semi-informative since

216 they only refer to third party responsibility for the sample collection procedures, without

217 mentioning any other description regarding the type of consent used (waived or presumed

218 consent included), or else when a reference to specific documents was made (e.g. certifications

219 or laws) but this was not easily readable/accessible. Nine answers (19.6\%) were not informative

220 because they were either left blank or referred to vague documents/criteria.

221 Concerning the strategies of collection and storage of biological samples, we found 24 biobanks

$222(52.2 \%)$ that do not accept samples from external research groups, with roughly similar

223 percentages in European (41.7\%) and American (50.0\%) continents. On the contrary, 22

224 biobanks (47.8\%) also store biological samples collected by external research groups. Sixteen of

225 them operate in Europe (72.7\%), 4 in America (18.2\%), 1 in Asia (4.5\%) and 1 in Oceania

226 (4.5\%). The majority of them (86.4\%), in order to accept samples for storage from external

227 groups, ask the latter to respect the same ethical requirements adopted by the biobank itself in its

228 sampling procedures. All the biobanks analysed make it possible for researchers to gain access to

229 their bio-specimen collection. Among them, only 2 biobanks [1 European (Estonia) and 1 
230 American (USA)] offer free and unconditioned accessibility to their samples, whereas the

231 remaining 95.7\% (44 out of 46) require specific conditions to be satisfied in order to give

232 permission to access their samples. However, our request for specifications regarding the

233 accessibility criteria (Question B4.1; see Figure 1) obtained only 12 informative answers in

234 which at least one criterion has been indicated. The analysis of these answers highlights how

235 sample accessibility seems to be linked to whether the applicants specify their research aims (e.g.

236 studies on a defined disease) and/or the origin of research funds (public, private or both). We

237 also considered those answers indicating that samples are for sale to be informative, or when one

238 of the above-listed criteria were specified and readable in external links provided in the answer.

239 Among the answers, 25 were classified as semi-informative. We defined the answers as semi-

240 informative when it was indicated that access is decided by third parties (e.g. IRBs, ethics

241 committees), when a vague criterion was stated (e.g. research project relevance, or researchers

242 working in the public interest), when specific agreements were indicated but the description was

243 difficult or impossible to read (i.e. in languages other than English or Italian), or when the

244 biobank institution had a person responsible for the access to biological samples. Finally, we

245 categorized 7 answers as not informative because they were left blank or because they refer to

246 agreements or documents that are totally inaccessible.

247 More than half of the biobanks (54.3\%) refers to a specific legal framework for access to their

248 biological samples. However, only 16 biobanks (34.8\%) provided informative answers showing

249 that there are no shared standards but different approaches influenced by the social context in

250 which they operate (Question B5.1; see Figure 1). The possibility to gain access to samples

251 seems to depend mainly on the approval of ethical committees, scientific bodies or bilateral

252 agreements (some biobanks also provided information regarding the model followed, e.g. OECD 
253 recommendations or legal contracts that concern customs laws regarding the commercial 254 circulation of biological materials). However, national laws (e.g., the Italian Garante della 255 Privacy, German Data Protection Laws, Spanish Act 14/2007 and Spanish Royal Decree 256 1716/2011), common international regulations (i.e. the European legal framework), or criteria 257 indicated in international agreements should also be taken into consideration when a request for 258 access to a collection of biological samples is presented. Concerning the possibility of finding 259 documents relative to the legal framework followed by the biobanks, only $31 \%$ provided detailed 260 information (Question B5.2; see Figure 1).

Data collection and accessibility: legal and ethical aspects

263 Most of the surveyed biobanks (41 out of 46;89.1\%) store data extracted from the analysis of 264 their own samples. Differently from what we observed for the biological samples, 23 out of 41 265 biobanks $(56.1 \%)$ also store data produced by external research groups that have used their 266 samples. Most of them (73.9\%) offers this service only if the external research groups follow the 267 same same legal framework of the biobank in question.

268 A slight difference between sample and data sharing propensity is evident when considering their 269 degree of accessibility. In fact, 3 biobanks [7.3\%; 2 Americans (USA) and 1 European 270 (Sweden)] do not allow any access to their data, whereas 3 other biobanks [7.3\%; 1 American 271 (USA) and 2 Europeans (France and Italy)] claim to follow a strict open data policy, giving 272 completely free access to their data. However, similarly for bio-specimens, the majority of 273 biobanks (35 out of 41; 85.4\%) allow external research groups to access their data in compliance 274 with certain conditions. We asked them which accessibility criteria they adopt (Question C2.1; 275 see Figure 1). Seven of these biobanks (20\%) gave us informative answers, describing 
276 codification procedures, providing reference to specific guidelines, giving access to projects

277 focused on specific groups of diseases, or stating clear criteria (e.g. co-authorship). Twenty of

278 the biobanks (57.1\%) provide semi-informative answers which refer to (i) third party

279 authorization for data access (i.e. ethics committees, IRBs, scientific boards); (ii) criteria linked

280 to the decision of a single researcher within the biobank institution; (ii) external documents that

281 do not clearly state the criteria adopted for data sharing; (iv) other vague criteria (e.g. application

282 for data access through a letter of intent). Finally, among the biobanks giving conditional access,

$2838(22.9 \%)$ were uninformative because their answers were inappropriate or because the criteria

284 were particularly vague (e.g. access given to authorized personnel, access given for research

285 made in public interest).

286 Similarly to what we observed for bio-specimens, also for data accessibility, the majority of the

287 biobanks (57.1\%) refer to a variety of legal frameworks, depending on the legislation of the 288 country where they mostly operate. Among the 35 biobanks (76.1\%) that grant conditional data 289 accessibility, 12 provided informative answers regarding this topic (Question C3.1; see Figure 290 1). They generally referred to national and international legal frameworks and agreements (e.g. 291 European legal framework, Material Transfer Agreement and the Health Information Privacy and 292 Portability Act in the USA). Interestingly, only one biobank highlighted the role of the privacy 293 guarantor for personal data protection in this procedure and only two biobanks (7.7\%) provided 294 the web link necessary to access to the legal framework documents (Question C3.2; see Figure 295 1). 296 297

\section{Discussion}


299 In this study, we explore how and at what level data and biological samples stored in research

300 biobanks are accessible and reusable. Primarily, most of the biobanks who responded to our

301 survey give access to their samples and data. However, free and unconditioned accessibility is

302 not common practice. In fact, external research groups eager to use biobank resources must

303 satisfy specific conditions in order to receive samples and gain access to databases.

304 Unfortunately, most of the biobanks we contacted provided vague or difficult-to-read

305 information about their accessibility criteria. This is an important result which shows that there is

306 still little clarity and a certain reluctance to share scientific resources. This lack of sharing

307 contrasts sharply with the emerging dependence of biomedical research on the activities of

308 biobanks (Kaye, 2011; Kaye et al., 2015). Nonetheless, this reluctance in sharing contradicts the

309 latest European research programme, Horizon 2020, where specific policies for open data and

310 open access are foreseen (Leonelli, Spichtinger \& Prainsack, 2015). Our analysis of the

311 informative answers points to three major issues related to the accessibility of biobank resources.

312 Firstly, applicants are requested to explain what they would like to do with the required

313 resources. This information is closely related to the specific data/bio-specimen sharing clause

314 contained in the original consent form. At the same time, it provides a certain degree of control

315 by the biobanks over the credentials and scientific reputation of the user and his research group.

316 Verifying the reliability and seriousness of applicants and minimizing the misuse of data and

317 samples is fundamental for biobanks. The ethical and technical approach of scientific-resource

318 management can promote public trust in the work of these institutions, thus increasing

319 willingness to participate in their activities (De Robbio, 2010).

320 Secondly, availability, amount and origin (public or private) of research funds are aspects

321 involved in the establishment of fruitful collaborations between biobanks and research labs. 
322 Publicly funded research seems to be preferred over studies granted by private (both profit and

323 non-profit-making) funding bodies. This result complies with the recommendation towards Open

324 Access of scientific resources produced with public funds proposed by the Organisation for

325 Economic Co-operation and Development (OECD) in its report "ECD principles and guidelines

326 for access to research data from public funding" published in 2007 (see Pilat \& Fukasaku, 2007).

327 The OECD report highlights the social, non epistemic, value of "public good" in sharing and the

328 importance of public scientific research and investment. However, only one of the biobanks

329 surveyed explicitly used the concept of sharing as "common good". On the other hand, scientific

330 resource sharing also has epistemic values regarding scientific rigor in favor of scientific

331 progress and this approach can guarantee a more "effective and transparent biobank practice"

332 (Demir \& Murtagh, 2013). Not only the source but also the availability of funds needed to carry

333 out the criteria research and payment for access to samples and data were found to be

334 fundamental criteria adopted by biobanks when deciding whether or not to make their data

335 available to third parties. In fact, the presence of clauses directly related to economic benefits for

336 biobanks reveals their possible "second nature" as profit-making institutions that offer services

337 concerning the collection and storage of biological samples and access to this material for

338 researchers. Thus, we can assume a relationship between private funds, buying and selling of

339 biobank resources and the widespread sharing of data and biological samples. However, it is

340 unclear if the commercial nature of biobanks is really a barrier to sharing. Caufield et al (2014)

341 suggest that the sharing of data and samples is a practice that "may be impacted or hindered by

342 the introduction of private funding and collaboration with private entities, as the expectations of

343 private entities and agreements governing such partnerships may create sharing barriers". Other

344 authors sustain that venture capitalism, with its continuously fluctuating expectations, have a 
345 strong impact on open data, above all when the boundaries between successful data sharing and

346 unfruitful initiatives become difficult to recognize, making financial investments, in these kinds

347 of scientific enterprise, risky but potentially rewarding (Leonelli, 2013).

348 Thirdly, we found that recognition of co-authorship is a requirement for some biobanks in order

349 to grant access to their data. Tenopir et al (2011), in their study on the data sharing practices and

350 perceptions of scientists, also reported the request for co-authorship on a publication as a fair

351 condition for the use of data produced by others. A similar result was also found by Milanovic,

352 Pontille and Cambon-Thomsen (2007) in their empirical study on the sharing of biological

353 samples and data in biosciences. This kind of request falls within the broader context of the

354 management of scientific resources in order to gain advantages in academic competition.

355 According to Vogeli et al (2006), this behavior may contribute to spreading a climate of mistrust

356 and lack of cooperation within the scientific community.

357 To sum up, these results suggest that economic and academic aspects are involved in 358 determining the extent of sharing of samples and data stored in biobanks. There is a consolidated

359 habit whereby biobank professionals mostly concentrate on commercial aspects whereas

360 researchers are more interested in academic pursuits (Pereira, 2013). Fortunately, these

361 detrimental attitudes for scientific progress and for the ethics of science cannot be generalized. In

362 fact, the culture of open science has begun to spread over the past decade in different fields of

363 life sciences (see Destro Bisol et al., 2014a; Destro Bisol et al., 2014b and related citations

364 therein). More specifically, scholars and researchers increasingly perceive the sharing of

365 scientific resources as a primary requirement for the development of new opportunities for

366 collaboration (e.g. see Foster \& Sharp, 2007; Fischer \& Zigmond, 2010; Boulton, et al 2012;

367 Mauthner \& Parry, 2013). In the case of research involving human subjects, data and sample 
368 sharing practices have been carried out following different protocols. All these protocols face

369 obstacles and restrictions due to both practical (e.g. the definition of informed consent) and

370 ethical issues [e.g. privacy and confidentiality concerns, prediction of potential reuses; see

371 Blumenthal et al., 2006; Teeters et al., 2008; Institute of Medicine (IOM), 2015]. Moreover,

372 given the different nature of data and samples, they do not necessarily follow identical sharing

373 procedures. In fact, while data sharing culture in biosciences seems to be catching on among

374 both researchers and policymakers, the same cannot be said for samples (Pereira, 2013).

375 However, Pereira (2013) depicts a more optimistic view about the willingness to share biological

376 resources by biobank professionals, highlighting that they "showed considerable interest in

377 advancing research and a generally altruistic perspective toward sharing samples and making

378 materials accessible to the research community". One good practice could consist in disclosing

379 the origin of funding and the aim of the research considering that today we are in an era in which

380 the characteristics of public research are ever more similar to those of private commercial 381 science (see Jasanoff, 2002; Krimsky, 2003; Krimsky, 2005) - e.g. openness and transparency of

382 claims and evidence substituted by secrecy (Ledford, 2014), fabrication of results and bias

383 against negative results (Fanelli, 2012). It is useful to remember that the 'bank' metaphor

384 overcomes the notion of "bio-repositories" or "bio-libraries" (Schneider, 2008) and that biobanks

385 can diverge diametrically in objectives and outcomes, or diametrically divergent visions and

386 practices can coexist within the same biobank. In this respect, biobanks are often regulated by

387 national and communitarian trade laws that hinder harmonization (i.e. bilateral agreements such

388 as the Transatlantic Trade and Investment Partnership between Europe and the USA) and this

389 could influence or divert local interests and national health services, as well as medical research

390 and local biotech companies. 
391 In the second step of our study, we investigated the reasons behind the observed high

392 heterogeneity of the requirements to gain access to the biobanks' resources. Most of the surveyed

393 biobanks adopted specific legal frameworks that researchers should take into consideration in

394 order to gain access to samples and data. The comparison of the information obtained from the

395 biobanks highlighted that these institutions follow different standards and procedures regarding

396 the sharing of their biological samples and data. The different modalities of resource accessibility

397 seem to be highly influenced by social context and legislations of the countries where the

398 biobanks operate. The fact that only few biobanks provided informative answers about this topic

399 could be interpretable as strong evidence that resource sharing is still a cumbersome practice.

400 This lack of clarity raises both ethical and practical issues: how to implement the sharing of

401 ethical conditions linked to the use of data and biological samples. A first practical step could be

402 the opportunity for donors to make their own choices through the informed consent process. The

403 ethical principles at the basis of informed consent in research involving human subjects (i.e.

404 respect, individual autonomy, protection of privacy) are inalienable and their importance is even

405 more evident in the case of biobanks due to their nature of institutions involving multiple

406 researchers within multiple research projects (Fullerton \& Lee, 2011). But, precisely due to their

407 nature, "it is difficult to obtain consent for all future research uses at the time of recruitment into

408 the biobank or before such research commences" (Kaye et al., 2015) a requirement specifically

409 stated in the seventh revision of the Declaration of Helsinki of 2013 (World Medical

410 Association, 2013). As declared by Jane Kaye et al. (2015), classical informed consent is an

411 inefficient tool in the attempt to overcome the obstacles in data and samples sharing due to its

412 static, paper-based format, which is generally only recognized at national level. Furthermore, we

413 must bear in mind that, particularly in the European context, privacy laws make the possibility to 
414 reuse data and samples extremely difficult. Interesting proposals coming from the Anglo-Saxon

415 world regard the possibility for participants to establish, through Information and

416 Communication Technologies (ICT), an ongoing, bidirectional communication with biobank

417 institutions to refresh or withdraw their consent for new research projects (Stein \& Terry, 2013;

418 Kaye et al., 2015). In such a dynamic form of consent, the authorization of individuals to handle

419 their personal data could travel with the same datasets containing biological and personal data

420 (Terry et al., 2013). The dynamic consent approach could be conceived as a way to preserve the

421 individual right to decide autonomously after having received detailed explanations about the

422 biobank sharing policies (participants could be provided with as much information as they want

423 concerning the aims of the projects aims and the methods used) and, at the same time, as a way

424 to protect individuals' privacy (each participant is free to handle and authorize flows of personal

425 data and to know regulations on data protection). Nevertheless, the dynamic consent approach

426 and strict laws on data protection are not useful in all cases. Indeed, privacy laws and the rising

427 attention towards individual rights can hinder the broad informed consent model and, overall,

428 can hinder those bio-repositories that have been established to protect collective rights such as

429 public health. For instance, cancer registries or retrospective studies could be damaged by the

430 strict rules on privacy proposed by the European Parliament resolution (12 March 2014) which

431 refers to the need to ask participants for their consent for every new research project involving

432 their data and samples (Casali, 2014). At European level, the ongoing discussion on consent

433 needed for medical research has involved most of the biobanks in which broad consent has been

434 used to involve participants. The proposed Data Protection Regulation, if transposed into law,

435 will constitute a challenge for biobanks and the scientific community will have to adapt to the

436 new European regulations (Hallinan \& Friedewald, 2015; Lucivero et al., 2015). On the one 
437 hand, the scientific community might use, when possible, ICTs to ask for an individual, specific

438 consent for every new project involving personal data. On the other hand, lawyers, policy makers

439 and experts might release the need of a specific consent, for research projects where open,

440 blanket or presumed consent has been used appropriately. This reasoning leads back to the

441 aforementioned problem of the lack of common and standardized operating procedures (SOPs)

442 and heterogeneity in access rules. In fact, this fragmentation not only limits the benefits of

443 sharing for the academia, but also contributes to increasing uncertainty of prospective donors in

444 deciding whether or not to give their contribution to the activities of biobanks. Undoubtedly,

445 progress in biomedical research is strictly linked to the involvement of the public in biobank

446 activities. In short, no donors, no biobanks. But the willingness of citizens to donate biological

447 samples and actively participate in biobank activities are in turn strictly linked to clarity when

448 explain the importance of participating in medical research (i.e. benefits deriving from biobank

449 research) and the manner in which biological samples and data will be used and made available

450 to the scientific community. In short, to foster mutually productive relationships among all the

451 stakeholders regarding biobanks, it is necessary to develop trust "understood as something which

452 demands knowledge and consent" (Richter, 2012), and to produce policies that make biobanks

453 trustworthy and sustainable institutions (Simeon-Dubach \& Watson, 2014).

\section{Conclusions}

458 In this paper, we have attempted to analyze the degree of accessibility and reusability of data and

459 biological samples stored in research biobanks following an empirical approach. Mainly, this 
460 study suggests that, in spite of general consensus of the scientific community concerning the

461 importance of open access of scientific resources, there are still sample and data sharing barriers

462 among biobanks and researchers. This does not mean that all these barriers should be necessarily

463 overcome thus leading to unrestricted access to biobank resources. In fact, some of these barriers

464 guarantee some fundamental rights of donors (e.g. privacy, misuse prevention) so should be

465 considered as "necessary". Therefore, bearing in mind the need to respect the donors" rights

466 when trying to overcome the sharing barriers, the accessibility of biological resources should not

467 be "unified" but rather go through standardized operating procedures.

468 Undoubtedly, this preliminary investigation needs to be continued and improved in order to

469 support (or even to question) the results obtained. Particularly, increasing the number of

470 surveyed biobanks and the related differences of socio-cultural contexts could help in producing

471 a more detailed picture of sharing behaviors and their differences related to the countries where

472 biobanks operate. Furthermore, more information could be obtained following a two-step

473 research protocol based on quantitative approaches such as those used in the present study, and a

474 second, more deeply focused, qualitative investigation (e.g. semi-structured interviews, focus

475 groups and interviews) into the main issues that emerge from the first step. According to Mertz

476 et al. (2014), empirical approaches provide an opportunity to overcome the classical descriptive

477 aim of social science methods applied in studying the scientific environment. Considering this

478 point of view, the so-called "empirical ethics" (see Hope, 1999 and Molewijk et al., 2004) may

479 contribute to increasing the knowledge on how and in what way all the agents involved in the life 480 cycle of biomedical research share their work. 


\section{Acknowledgements}

484 We wish to thank the biobanks and their anonymous members who contributed to the survey. We

485 would also like to thank the reviewers for their helpful comments and suggestions that greatly 486 contributed to improving the final version of the manuscript. 


\section{References}

489

490 Arzberger P, Schroeder P, Beaulieu A, Bowker G, Casey K, Laaksonen L, Moorman D, Uhlir P, 491 Wouters P. 2004. Promoting access to public research data for scientific, economic and social 492 development. Data Science Journal 3:135-152. DOI: 10.2481/dsj.3.135.

493

494 Blumenthal D, Campbell EG, Gokhale M, Yucel R, Clarridge B, Hilgartner S, Holtzman NA. 495 2006. Data withholding in genetics and the other life sciences: prevalences and predictors. 496 Academic Medicine 81:137-145. DOI: 10.1097/00001888-200602000-00008.

497

498

Boulton G, Campbell P, Collins B, Elias P, Hall W, Laurie G, O’Neill BA, Rawlins M, Thornton 499 DJ, Vallance P, Walport M. 2012. Science as an open enterprise. London: The Royal Society.

501 Cadigan RJ, Juengst E, Davis A, Henderson G. 2014. Underutilization of specimens in biobanks: 502 an ethical as well as a practical concern?. Genetics in Medicine 16:738-740. DOI: 503 10.1038/gim.2014.38.

504

505 Casali PG. 2014. Risks of the new EU data protection regulation: an ESMO position paper 506 endorsed by the European oncology community. Annals of Oncology 25:1458-1461. DOI: 507 10.1093/annonc/mdu218.

508

509 Caulfield T, Burningham S, Joly Y, Master Z, Shabani M, Borry P, Becker A, Burgess M, Calder

510 K, Critchley C, Edwards K, Fullerton SM, Gottweis H, Hyde-Lay R, Illes J, Isasi R, Kato K, 
511 Kaye J, Knoppers B, Lynch J, McGuire A, Meslin E, Nicol D, O’Doherty K, Ogbogu U,

512 Otlowski M, Pullman D, Ries N, Scott C, Sears M, Wallace H, Zawati MH. 2014. A review of

513 the key issues associated with the commercialization of biobanks. Journal of Law and the

514 Biosciences 1:94-110. DOI: 10.1093/jlb/1st004.

515

516 Colledge F, Persson K, Elger B, Shaw D. 2014. Sample and data sharing barriers in biobanking:

517 consent, committees, and compromises. Annals of Diagnostic Pathology 18:78-81. DOI:

518 10.1016/j.anndiagpath.2013.12.002.

519

520 D’Abramo F. 2015. Biobank research, informed consent and society. Towards a new alliance?.

521 Journal of Epidemiology and Community Health [Epub ahead of print]. DOI: 10.1136/jech522 2014-205215.

523

524 D’Abramo F, Schildmann J, Vollmann J. 2015 Research participants' perceptions and views on 525 consent for biobank research: a review of empirical data and ethical analysis. BMC Medical 526 Ethics 16:60. DOI: 10.1186/s12910-015-0053-5.

De Robbio A. 2010. Biobanche e proprietà intellettuale: commons o caveau?. Bibliotime 14:3.

529

530 Demir I, Murtagh MJ. 2013. Data sharing across biobanks: epistemic values, data mutability and 531 data incommensurability. New Genetics and Society 32:350-365. DOI: $10.1080 / 14636778.2013 .846582$. 
534 Destro Bisol G, Anagnostou P, Bruner E, Capocasa M, Canali S, Danubio ME, Di Vincenzo F,

535 Fantini B, Greco P, Moggi Cecchi J, Parenti F, Pavanello M, Pettener D, Pievani T, Saracino B,

536 Rufo F, Sanna E, Vargiu R, Vona G. 2014a. Open data, Science and Society: launching Oasis,

537 the flagship initiative of the Istituto Italiano di Antropologia. Journal of Anthropological 538 Sciences 92:I-IV. DOI: 10.4436/JASS.92016.

539

540 Destro Bisol G, Anagnostou P, Capocasa M, Bencivelli S, Cerroni A, Contreras J, Enke N,

541 Fantini B, Greco P, Heeney C, Luzi D, Manghi P, Mascalzoni D, Molloy J, Parenti F, Wicherts J,

542 Boulton G. 2014b. Perspectives on open science and scientific data sharing: an interdisciplinary 543 workshop. Journal of Anthropological Sciences 92:179-200. DOI: 10.4436/JASS.92006.

545 Fanelli D. 2012. Negative results are disappearing from most disciplines and countries. 546 Scientometrics 90:891-904. DOI: 10.1007/s11192-011-0494-7.

547

548 Fischer BA, Zigmond MJ. 2010. The essential nature of sharing in science. Science and 549 Engineering Ethics 16:783-799. DOI: 10.1007/s11948-010-9239-x.

551 Foster MW, Sharp RR. 2007. Share and share alike: deciding how to distribute the scientific and 552 social benefits of genomic data. Nature Reviews Genetics 8:633-639. DOI: 10.1038/nrg2124.

554 Fullerton SM, Lee SS. 2011. Secondary uses and the governance of de-identified data: lessons 555 from the human genome diversity panel. BMC Medical Ethics 12:16. DOI: 10.1186/1472-6939$55612-16$. 
558 Godard B, Ozdemir V, Fortin M, Égalité N. 2010. Ethnocultural community leaders' views and

559 perceptions on biobanks and population specific genomic research: a qualitative research study.

560 Public Understanding of Science 19:469-485. DOI: 10.1177/0963662509104721.

561

562 Haga SB, Beskow LM. 2008. Ethical, legal, and social implications of biobanks for genetics 563 research. Advances in Genetics 60:505-544. DOI: 10.1016/S0065-2660(07)00418-X.

565 Hallinan D, Friedewald M. 2015. Open consent, biobanking and data protection law: can open

566 consent be 'informed' under the forthcoming data protection regulation? Life Sciences, Society 567 and Policy 11:1. DOI: 10.1186/s40504-014-0020-9.

568

569 Henderson GE, Cadigan RJ, Edwards TP, Conlon I, Nelson AG, Evans JP, Davis AM, Zimmer 570 C, Weiner BJ. 2013. Characterizing biobank organizations in the U.S.: results from a national 571 survey. Genome Medicine 5:3. DOI: 10.1186/gm407.

572

573 Hirtzlin I, Dubreuil C, Preaubert N, Duchier J, Jansen B, Simon J, Lobato De Faria P, Perez574 Lezaun A, Visser B, Williams GD, Cambon-Thomsen A; EUROGENBANK Consortium. 2003. 575 An empirical survey on biobanking of human genetic material and data in six EU countries. 576 European Journal of Human Genetics 11:475-488.

577

578 Hope T. 1999. Empirical medical ethics. Journal of Medical Ethics 25:219. DOI: $57910.1136 /$ jme.25.3.219. 
581 Institute of Medicine (IOM). 2015. Sharing clinical trial data: maximizing benefits, minimizing 582 risk. Washington: The National Academies Press.

583

584 Jasanoff S. 2002. The life sciences and the rule of law. Journal of Molecular Biology 319:891585 899. DOI: 10.1016/S0022-2836(02)00337-6.

586

587 Kaufman DJ, Murphy-Bollinger J, Scott J, Hudson KL. 2009. Public opinion about the 588 importance of privacy in biobank research. American Journal of Human Genetics 85:643-654. 589 DOI: 10.1016/j.ajhg.2009.

590

591 Kaye J. 2006. Do we need a uniform regulatory system for biobanks across Europe?. European 592 Journal of Human Genetics 14:245-248. DOI: 10.1038/sj.ejhg.5201530.

593

594 Kaye J. 2011. From single biobanks to international networks: developing e-governance. Human 595 Genetics 130:377-382. DOI: 10.1007/s00439-011-1063-0.

596

597 Kaye J. 2012. The tension between data sharing and the protection of privacy in genomics 598 research. Annual Review of Genomics and Human Genetics 13:415-431. DOI: 10.1146/annurev599 genom-082410-101454.

600 
601 Kaye J, Whitley EA, Lund D, Morrison M, Teare H, Melham K. 2015. Dynamic consent: a 602 patient interface for twenty-first century research networks. European Journal of Human 603 Genetics 23:141-146. DOI: 10.1038/ejhg.2014.71.

604

605 Knoppers BM. 2010. Consent to 'personal' genomics and privacy. EMBO Reports 11:416-419.

606 DOI: 10.1038/embor.2010.69.

607

608 Krimsky S. 2003. Science in the private interest: has the lure of profits corrupted biomedical 609 research? Lanham: Rowman and Littlefield.

610

611 Krimsky S. 2005. The funding effect in science and its implications for the judiciary. Journal of 612 Law and Policy 13:46-68.

613

614 Ledford H. 2014. FDA debates trial-data secrecy. Nature 511:519. DOI: 10.1038/511519a.

615

616 Lemke AA, Wolf WA, Hebert-Beirne J, Smith ME. 2010. Public and biobank participant 617 attitudes toward genetic research participation and data sharing. Public Health Genomics 13:368618 377. DOI: $10.1159 / 000276767$.

619

620 Leonelli S. 2013. Why the current insistence on Open Access to scientific data? Big data, 621 knowledge production, and the political economy of contemporary biology. Bulletin of Science, 622 Technology \& Society 33:6-11. DOI: 10.1177/0270467613496768.

623 
624 Leonelli S, Spichtinger D, Prainsack B. 2015. Sticks and carrots: encouraging open science at its 625 source. Geography and Environment 2:12-16. DOI: 10.1002/geo2.2.

626

627 Lucivero F, Del Savio L, Aicardi C, Dove ES, Marris C, Prainsack B, Saleem N, Tempini N, 628 Turrini M. 2015. A response to the WMA draft declaration on ethical considerations regarding 629 health databases and biobanks. DOI: 10.13140/RG.2.1.3334.4163.

630

631 Macilotti M. 2013. Informed consent and research biobanks: a challenge in three dimensions. In:

632 Pascuzzi G, Izzo U, Macilotti M, eds. Comparative issues in the governance of research 633 biobanks. Berlin Heidelberg: Springer, 143-161. DOI: 10.1007/978-3-642-33116-9_9.

634

635 Mauthner NS, Parry O. 2013. Open Access digital data sharing: principles, policies and 636 practices. Social Epistemology 27:47-67. DOI: 10.1080/02691728.2012.760663.

637

638 Mertz M, Inthorn J, Renz G, Rothenberger LG, Salloch S, Schildmann J, Wöhlke S, Schicktanz 639 S. 2014. Research across the disciplines: a road map for quality criteria in empirical ethics 640 research. BMC Medical Ethics 15:17. DOI: 10.1186/1472-6939-15-17.

641

642 Milanovic F, Pontille D, Cambon-Thomsen A. 2007. Biobanking and data sharing: a plurality of 643 exchange regimes. Genomics, Society \& Policy 3:17-30. DOI: 10.1186/1746-5354-3-1-17. 
645 Milia N, Congiu A, Anagnostou P, Montinaro F, Capocasa M, Sanna E, Destro Bisol G. 2012.

646 Mine, yours, ours? Sharing data on human genetic variation. PLoS One 7:e37552. DOI:

647 10.1371/journal.pone.0037552.

648

649 Molewijk B, Stiggelbout AM, Otten W, Dupuis HM, Kievit J. 2004. Scientific contribution. 650 Empirical data and moral theory. A plea for integrated empirical ethics. Medicine, Health Care 651 and Philosophy 7:55-69. DOI: 10.1023/B:MHEP.0000021848.75590.b0.

652

653 Nelson B. 2009. Empty archives. Nature 461:160-163. DOI: 10.1038/461160a.

654

655 Oliver JM, Slashinski MJ, Wang T, Kelly PA, Hilsenbeck SH, McGuire AL. 2012. Balancing the 656 risks and benefits of genomic data sharing: genome research participants' perspectives. Public 657 Health Genomics 15:106-114. DOI: 10.1159/000334718.

658

659 Pereira S. 2013. Motivations and barriers to sharing biological samples: a case study. Journal of 660 Personalized Medicine 3:102-110. DOI: 10.3390/jpm3020102.

661

662 Pilat D, Fukasaku Y. 2007. OECD principles and guidelines for access to research data from 663 public funding. Data Science Journal 6:OD4-OD11. DOI: 10.2481/dsj.6.OD4.

664

665 Richter C. 2012. Biobanking. Trust as basis for responsibility. In: Dabrock P, Taupitz J, Ried J, 666 eds. Trust in biobanking. Dealing with ethical, legal and social issues in an emerging field of 667 biotechnology. Berlin-Heidelberg: Springer, 43-66. 
668

669 Schneider I. 2008. 'This is not a national biobank...': the politics of local biobanks in Germany.

670 In: Gottweis H, Petersen A, eds. Biobanks: governance in comparative perspective. Abingdon:

671 Routledge, 88-108.

672

673 Simeon-Dubach D, Watson P. 2014. Biobanking 3.0: evidence based and customer focused

674 biobanking. Clinical Biochemistry 47:300-308. DOI: 10.1016/j.clinbiochem.2013.12.018.

675

676 Stein DT, Terry SF. 2013. Reforming biobank consent policy: a necessary move away from

677 broad consent toward dynamic consent. Genetic Testing and Molecular Biomarkers 17:855-856.

678 DOI: $10.1089 /$ gtmb.2013.1550.

679

680 Teeters JL, Harris KD, Millman KJ, Olshausen BA, Sommer FT. 2008. Data sharing for 681 computational neuroscience. Neuroinformatics 6:47-55. DOI: 10.1007/s12021-008-9009-y. 682

683 Tenopir C, Allard S, Douglass K, Aydinoglu AU, Wu L, Read E, Manoff M, Frame M. 2011. 684 Data sharing by scientists: practices and perceptions. PLoS One 6:e21101. DOI: 685 10.1371/journal.pone.0021101.

686

687 Terry SF, Shelton R, Biggers G, Baker D, Edwards K. 2013. The haystack is made of needles. 688 Genetic Testing and Molecular Biomarkers 17:175-177. DOI: 10.1089/gtmb.2012.1542. 689 
690 Trinidad SB, Fullerton SM, Bares JM, Jarvik GP, Larson EB, Burke W. 2010. Genomic research 691 and wide data sharing: views of prospective participants. Genetics in Medicine 12:486-495. DOI: 692 10.1097/GIM.0b013e3181e38f9e.

693

694 Vogeli C, Yucel R, Bendavid E, Jones LM, Anderson MS, Louis KS, Campbell EG. 2006. Data 695 withholding and the next generation of scientists: results of a national survey. Academic 696 Medicine 81:128-136. DOI: 10.1097/00001888-200602000-00007.

697

698 Wolf SM, Crock BN, Van Ness B, Lawrenz F, Kahn JP, Beskow LM, Cho MK, Christman MF, 699 Green RC, Hall R, Illes J, Keane M, Knoppers BM, Koenig BA, Kohane IS, Leroy B, Maschke 700 KJ, McGeveran W, Ossorio P, Parker LS, Petersen GM, Richardson HS, Scott JA, Terry SF, 701 Wilfond BS, Wolf WA. 2012. Managing incidental findings and research results in genomic 702 research involving biobanks and archived data sets. Genetics in Medicine 14:361-384. DOI: 703 10.1038/gim.2012.23.

704

705

World Medical Association 2013. World Medical Association Declaration of Helsinki. Ethical 706 principles for medical research involving human subjects. Journal of the American Medical 707 Association 310:2191-2194. DOI: 10.1001/jama.2013.281053.

708

709 Zika E, Paci D, Braun A, Rijkers-Defrasne S, Deschenes M, Fortier I, Laage-Hellman J, Scerri

710 CA, Ibarreta D. 2011. A European survey on biobanks: trends and issues. Public Health

711 Genomics 14:96-103. DOI: 10.1159/00029627.

712

713 


\section{Table $\mathbf{1}$ (on next page)}

Geographic distribution of biobanks involved in this study.

Geographic distribution of biobanks involved in this study. Percentage of respondents in brackets. 


\begin{tabular}{|c|c|c|c|}
\hline \multirow[b]{2}{*}{ Continent } & \multirow[b]{2}{*}{ Country } & \multicolumn{2}{|c|}{ Biobanks } \\
\hline & & Invited & Responded \\
\hline \multirow{2}{*}{ Africa } & South Africa & 1 & $0(0)$ \\
\hline & Zimbabwe & 1 & $0(0)$ \\
\hline \multirow{3}{*}{ America } & Brazil & 1 & $0(0)$ \\
\hline & Canada & 12 & $2(16.7)$ \\
\hline & USA & 91 & $14(15.4)$ \\
\hline \multirow{11}{*}{ Asia } & China & 2 & $0(0)$ \\
\hline & India & 3 & $0(0)$ \\
\hline & Iran & 1 & $0(0)$ \\
\hline & Israel & 3 & $1(33.3)$ \\
\hline & Japan & 4 & $1(25.0)$ \\
\hline & Korea & 1 & $0(0)$ \\
\hline & Malaysia & 3 & $0(0)$ \\
\hline & Qatar & 1 & $0(0)$ \\
\hline & Singapore & 4 & $0(0)$ \\
\hline & Taiwan & 1 & $0(0)$ \\
\hline & Thailand & 2 & $0(0)$ \\
\hline \multirow{22}{*}{ Europe } & Austria & 4 & $2(50.0)$ \\
\hline & Belgium & 3 & $1(33.3)$ \\
\hline & Estonia & 1 & $1(100)$ \\
\hline & Finland & 1 & $1(100)$ \\
\hline & France & 7 & $2(28.6)$ \\
\hline & Germany & 19 & $3(15.8)$ \\
\hline & Greece & 2 & $0(0)$ \\
\hline & Hungary & 1 & $0(0)$ \\
\hline & Iceland & 1 & $0(0)$ \\
\hline & Ireland & 3 & $1(33.3)$ \\
\hline & Italy & 12 & $5(41.7)$ \\
\hline & Latvia & 1 & $0(0)$ \\
\hline & Luxembourg & 1 & $0(0)$ \\
\hline & Malta & 1 & $0(0)$ \\
\hline & Netherlands & 4 & $1(25.0)$ \\
\hline & Norway & 2 & $1(50.0)$ \\
\hline & Poland & 1 & $0(0)$ \\
\hline & Portugal & 1 & $0(0)$ \\
\hline & Spain & 5 & $1(20.0)$ \\
\hline & Sweden & 5 & $1(20.0)$ \\
\hline & Switzerland & 4 & $0(0)$ \\
\hline & United Kingdom & 16 & $6(37.5)$ \\
\hline Oceania & Australia & 12 & $2(16.7)$ \\
\hline & Total & 238 & $46(19.3)$ \\
\hline
\end{tabular}


1

Informativeness of the answers given to the open questions.

Informativeness of the answers given to the open questions.

B2. Which are the ethical requirements followed by the biobank in the sample collection procedure?

B4.1 If sample are conditionally accessible, which are the accessibility criteria adopted by the biobank?

B5.1 If yes, which is this legal framework?

B5.2 If yes, where it is possible to find it?

C2.1 If data are conditionally accessible, which are the accessibility criteria adopted by the biobank?

C3.1 If yes, which is this legal framework?

C3.2 If yes, where it is possible to find it?

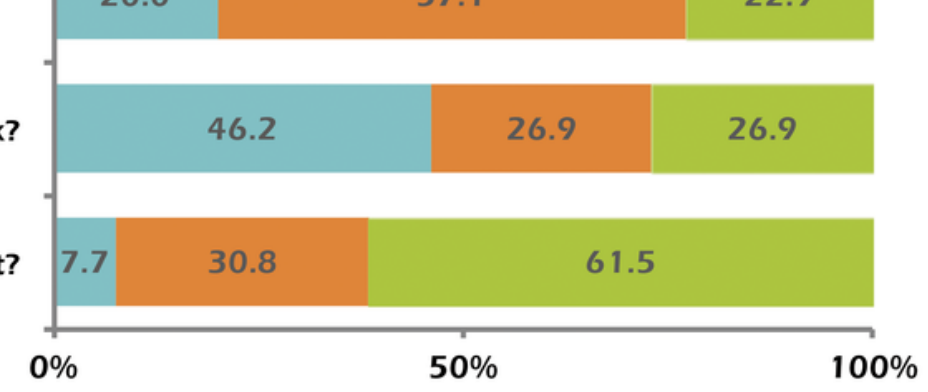

\section{8}

32.6

19.6

27.3

56.8

15.9

-

\section{3}

33.3

13.3

\section{0}

44.8

24.1

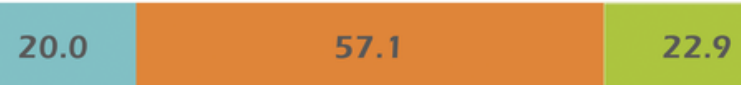

Informative Semi-Informative Not Informative/No answer 